\title{
SQUARING DOWN DESIGN FOR OVER-ACTUATED SYSTEMS IN MULTIVARIABLE QFT
}

\author{
Edward Boje \\ School of Electrical, Electronic and Computer Engineering \\ University of KwaZulu-Natal, 4041, Durban, South Africa \\ email: boje@ukzn.ac.za
}

\begin{abstract}
This paper discusses the problem of design of a control authority allocation to "square down" uncertain, over-actuated systems before feedback design is contemplated. It is assumed that the uncertainty in the plant is unstructured and that the subsequent design will be based on Horowitz' quantitative feedback theory (QFT).

Copyright (C) 2005 IFAC.
\end{abstract}

Keywords: Robust Control, Quantitative Feedback Theory, Over-Actuated, Non-Square Systems.

\section{INTRODUCTION}

Multivariable QFT is an established method for feedback design of uncertain multivariable systems. Like all methods for robust multivariable control design, MIMO QFT suffers from certain limitations, including over-design and the requirement for a certain level of expertise in design execution. This notwithstanding, the method is an attractive option for practical design and tuning, where for example, plant model uncertainty comes in a complicated way from uncertainty in underlying parameters of the plant or may be the outcome of repeated system identification experiments.

This paper addresses the problem of how to allocate controller outputs to plant inputs (i.e. actuator authority allocation) in the case of non-square, overactuated plants. Consider the plant illustrated in Figure 1 where $\mathrm{P}(\mathrm{s})$ is full rank (except at its transmission zeros) with $m$ inputs and $n$ outputs. Clearly, the loop transfer function must be square $(n \times n)$ and a $(m \times n)$ pre-compensator $\boldsymbol{K}(s)$ will be designed before a diagonal controller design. For simplicity assume that the sensor has high bandwidth relative to the loop bandwidth, $\boldsymbol{H}=\boldsymbol{I}$. For this problem, a number of considerations may be important:

1) To minimise over-design caused by the design method. MIMO QFT uses over-bounding of yet to be designed loops to enable a sequential design procedure. Inherently, this approach may be conservative but approximate decoupling can reduce the level of over design.

2) To make use of actuators within their bandwidth and amplitude capability.

3) To design with integrity against actuator and sensor failure.

The general approach of all MIMO QFT methods can be summarised as that of finding appropriate matrix manipulations and splittings to separate the plant and a diagonal controller in such a way that over design is minimised (Boje, 2002). For non-diagonal controller design, it is convenient both from a design perspective and for final tuning, to separate a lowgain non-diagonal part of the controller from the diagonal part and this paper seeks to extend the existing approach to this problem (Boje and Nwokah, 2001, and Boje, 2002) to non-square plants. In previous work, the plant matrix inverse was used as the plant transfer function was taken to be square and regular. The control authority pre-compensator was designed as a set of dynamic elementary operations,

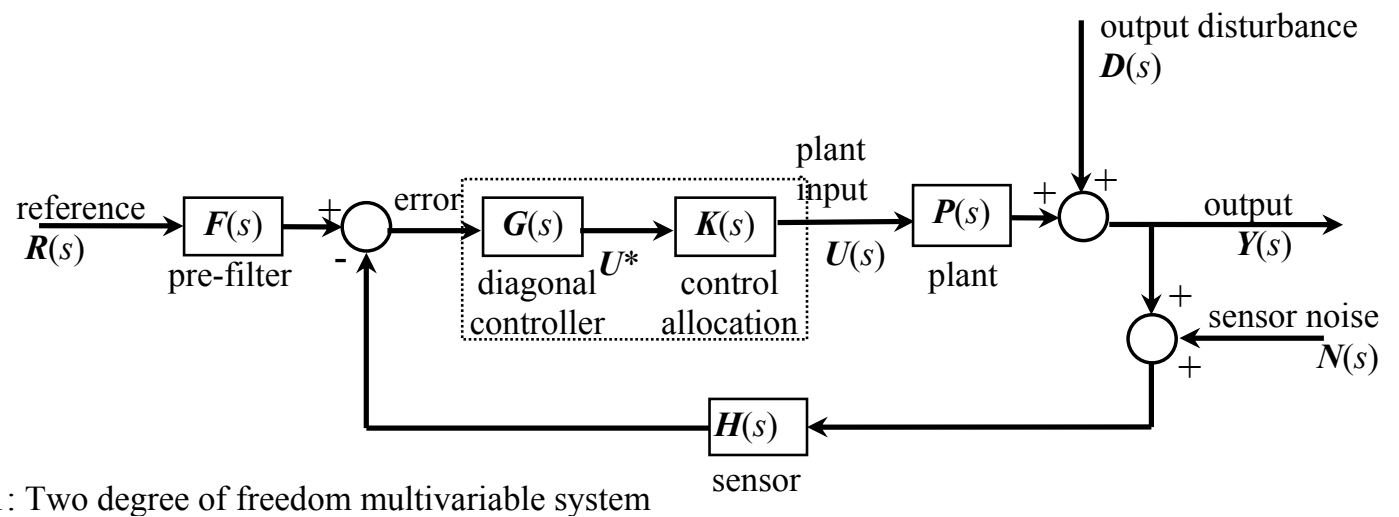

Figure 1: Two degree of freedom multivariable system 
ensuring that the designer could keep track of the gain of the pre-compensator and ensuring that it did not add right hand plant poles and zeros to the loop transmission. For non-square, over-actuated plants, it is not possible to work with the plant inverse (unless the control authority allocation is assigned) and a non plant inverting approach may be required.

The focus of this paper is to develop a control allocation design method that aims to reduce the interaction at appropriate frequencies. In general, this will ease the problem of over-design inherent in the MIMO QFT method and may allow decentralised stability results to be applied. The design must be tractable when applied to system with practical uncertainty (for example described by an enumerated plant set). Other approaches to the squaring-down problem have investigated a single plant instance and use the squaring-down to influence (where possible) the transmission zeros (especially those in the right hand plane) of the resulting square system.

\section{CONTROL AUTHORITY DESIGN}

2.1 Revision of design method with plant inversion and diagonal matrix splitting

The transfer function from reference to output is, $\boldsymbol{T}_{Y / R}=(\boldsymbol{I}+\boldsymbol{P} \boldsymbol{K} \boldsymbol{G})^{-1} \boldsymbol{P} \boldsymbol{K} \boldsymbol{G} \boldsymbol{F}$, and separating the diagonal controller from the remainder of the loop transfer function, this can be written

$$
\left((\boldsymbol{P K})^{-1}+\boldsymbol{G}\right) \boldsymbol{T}_{Y / R}=\boldsymbol{G F}
$$

Performing an appropriate splitting of diagonal $(\operatorname{diag}\{\cdot\})$ and off-diagonal $(\operatorname{od}\{\cdot\})$ parts yields,

$$
\left(\operatorname{diag}\left\{(\boldsymbol{P K})^{-1}\right\}+\boldsymbol{G}\right)\left(\boldsymbol{I}+\boldsymbol{X}_{\text {inv }}\right) \boldsymbol{T}_{Y / R}=\boldsymbol{G} \boldsymbol{F}
$$

with the interaction matrix (with subscript inv to denote that it is based on plant inversion),

$$
\begin{aligned}
\boldsymbol{X}_{\text {inv }} & =\left(\operatorname{diag}\left\{(\boldsymbol{P K})^{-1}\right\}+\boldsymbol{G}\right)^{-1} \operatorname{od}\left\{(\boldsymbol{P K})^{-1}\right\} \\
& =\boldsymbol{S}_{\text {diag_inv }} \operatorname{diag}\left\{(\boldsymbol{P K})^{-1}\right\}^{-1} \operatorname{od}\left\{(\boldsymbol{P K})^{-1}\right\}
\end{aligned}
$$

In previous work (Limebeer, 1982; Nwokah, Nordgren and Grewal, 1995; Skogestad and Postlethwaite, 1996; Boje and Nwokah, 2001; and Boje, 2002) the role of the interaction matrix has been recognised: If the spectral radius of the interaction matrix is less than unity $\left(\rho\left\{\boldsymbol{X}_{\text {inv }}\right\} \leq 1\right)$ for all frequencies, $\Delta \arg \left\{\boldsymbol{I}+\boldsymbol{X}_{\text {inv }}\right\}=0$ around the Nyquist contour and closed loop stability is determined by the behaviour of the diagonal elements of the design. Secondly, small interaction matrix elements contribute to small over-design as the QFT design is executed by writing,

$$
\boldsymbol{T}_{Y / R}=\boldsymbol{T}_{\text {diag_inv }} \boldsymbol{F}-\boldsymbol{X}_{\text {inv }} \boldsymbol{T}_{Y / R}
$$

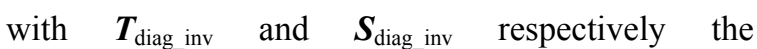
complimentary sensitivity and sensitivity of the diagonal loop using the inverse of the plant with control allocation. In eq.(3) it is readily observed that the interaction matrix can be made small either by small sensitivity (typically at low frequency relative to the loop bandwidth where the loop gain can be made large), or by appropriate control authority allocation making $\operatorname{diag}\left\{(\boldsymbol{P K})^{-1}\right\}^{-1} \operatorname{od}\left\{(\boldsymbol{P K})^{-1}\right\}$ small. Previous work has used the Perron root of the interaction matrix, $\lambda_{P}=\lambda_{\max }\{|\boldsymbol{X}|\} \leq \rho\{\boldsymbol{X}\}$, with element-wise magnitudes. The benefit of using the Perron root is that for irreducible matrices it is a monotonic, differentiable function of the matrix elements and reducing the magnitude of any element will reduce the Perron root. The eigenvalue sensitivity can be obtained from the participation factor - the normalised product of the corresponding right and left Perron root eigenvectors.

When $\boldsymbol{P}$ is square and regular, $(\boldsymbol{P K})^{-1}=\boldsymbol{K}^{-1} \boldsymbol{P}^{-1}$ and $\boldsymbol{K}$ can be designed via its inverse as a set of elementary operations and the inverse of the resulting unimodular matrix is easy to compute. This approach has connections to early work of Rosenbrock (1969) on the inverse Nyquist array.

\subsection{Tracking design without plant inversion}

For non-square $\boldsymbol{P}$, it is necessary to have an estimate of the pre-filter, $\boldsymbol{F}$, or equivalently, specifications on the complimentary sensitivity, $\boldsymbol{T}$ in order to obtain non-plant inversion formulas as follows

$$
\begin{gathered}
\boldsymbol{T}=\boldsymbol{T}_{Y / R} \boldsymbol{F}^{-1}=(\boldsymbol{I}+\boldsymbol{P} \boldsymbol{K} \boldsymbol{G})^{-1} \boldsymbol{P} \boldsymbol{K} \boldsymbol{T} \\
\boldsymbol{T}\left(\boldsymbol{G}^{-1}+\boldsymbol{P K}\right)=\boldsymbol{P} \boldsymbol{K} \\
\boldsymbol{T}\left(\boldsymbol{G}^{-1}+\operatorname{diag}\{\boldsymbol{P K}\}\right)+(\boldsymbol{I}-\boldsymbol{S}) \operatorname{od}\{\boldsymbol{P K}\}=\boldsymbol{P} \boldsymbol{K} \\
\boldsymbol{T}=\left(\boldsymbol{I}+\boldsymbol{S} \text { od }\{\boldsymbol{P K}\} \operatorname{diag}\{\boldsymbol{P K}\}^{-1}\right) \boldsymbol{T}_{\operatorname{diag}} \\
\boldsymbol{T}=(\boldsymbol{I}+\boldsymbol{X}) \boldsymbol{T}_{\operatorname{diag}} \\
\boldsymbol{X}=\boldsymbol{S} \text { od }\{\boldsymbol{P K}\} \operatorname{diag}\{\boldsymbol{P K}\}^{-1}
\end{gathered}
$$

With $(\boldsymbol{I}+\boldsymbol{Z})=(\boldsymbol{I}+\boldsymbol{X})^{-1}$, eq.(5) can be written $(\boldsymbol{I}+\boldsymbol{Z}) \boldsymbol{T}=\boldsymbol{T}_{\text {diag }} . \quad$ If $\quad \rho\{\boldsymbol{X}\} \leq 1, \quad(\boldsymbol{I}+\boldsymbol{X})^{-1}=\sum_{k=0}^{\infty} \boldsymbol{X}^{k}$ converges and the spectral radius of $\boldsymbol{Z}$ is bounded by,

$$
\rho\{\boldsymbol{Z}\}=\rho\left\{\sum_{k=1}^{\infty} \boldsymbol{X}^{k}\right\} \leq \sum_{k=1}^{\infty}\left(\rho\{\boldsymbol{X}\}^{k}\right)=\frac{\rho\{\boldsymbol{X}\}}{1-\rho\{\boldsymbol{X}\}}
$$

The Perron root can also be over-bounded by,

$$
\begin{aligned}
& \lambda_{P}\{\boldsymbol{Z}\}=\lambda_{P}\left\{\left|\sum_{k=1}^{\infty} \boldsymbol{X}^{k}\right|\right\} \leq \sum_{k=1}^{\infty}\left(\lambda_{P}\{\boldsymbol{X}\}\right)^{k}=\frac{\lambda_{P}\{\boldsymbol{X}\}}{1-\lambda_{P}\{\boldsymbol{X}\}} \\
& \lambda_{P}\{\boldsymbol{X}\} \leq 1 / 2 \Rightarrow \lambda_{P}\{\boldsymbol{Z}\} \leq 1 \Rightarrow \rho\{\boldsymbol{Z}\} \leq 1
\end{aligned}
$$

Theorem 1: Decentralised stability

Given:

a) $\quad \boldsymbol{P} \in\{\boldsymbol{P}\}$, regular, having $N_{\mathrm{PO}}$ (open loop) poles and $N_{\text {ZO }}$ (open loop transmission) zeros in the closed right half plane (RHP), and no hidden unstable modes.

b) $(\boldsymbol{I}+\boldsymbol{P K} \boldsymbol{G})^{-1} \boldsymbol{P}$ analytic at the closed RHP poles of $\mathbf{P}$ (including infinity).

c) $\boldsymbol{F}, \boldsymbol{K}$, and $\boldsymbol{G}$ stable and $\boldsymbol{K} \boldsymbol{G}$ minimum phase by design.

d) $\rho(\boldsymbol{X})<1$ for all $\boldsymbol{P} \in\{\boldsymbol{P}\}$ and for $\mathrm{s} \in \delta \mathrm{D}$, the Nyquist contour covering the RHP. 
Then, $\boldsymbol{T}$ in eq(5) is stable iff each diagonal loop, $\ell_{i}=g_{i}(\boldsymbol{P K})_{i i}$ is designed so that, for all $\boldsymbol{P} \in\{\boldsymbol{P}\}$, the loci of $1 / \ell_{i}$, taken together, encircle the $(-1, j 0)$ point $N_{\mathrm{D}}=-N_{\mathrm{ZO}}$ times (in the opposite direction) as $s$ traverses the Nyquist contour.

Proof: Following Maciejowski (1989), Theorem 2.8, Let $(\boldsymbol{I}+\boldsymbol{P K G})$ have $N_{\mathrm{CL}}$ RHP zeros. $\boldsymbol{P}$ has $N_{\mathrm{PO}} \mathrm{RHP}$ poles and $N_{\text {ZO }}$ RHP zeros. (c) $\Rightarrow \Delta \arg \{\operatorname{det}(\boldsymbol{G})\}=0$. (d) $\rho(\boldsymbol{X})<1 \Rightarrow \Delta \arg \{\operatorname{det}(\boldsymbol{I}+\boldsymbol{X})\}=0$. Now write, $(\boldsymbol{I}+\boldsymbol{P} \boldsymbol{K} \boldsymbol{G})(\boldsymbol{I}+\boldsymbol{X})=\boldsymbol{P} \boldsymbol{K}\left(\operatorname{diag}\{\boldsymbol{P K}\}^{-1}+\boldsymbol{G}\right)$ and apply the Principle of Argument around the Nyquist contour,

$$
\begin{aligned}
& \Delta \arg \{\operatorname{det}(\boldsymbol{I}+\boldsymbol{P} \boldsymbol{K} \boldsymbol{G})\}+\Delta \arg \{\operatorname{det}(\boldsymbol{I}+\boldsymbol{X})\} \\
& =\Delta \arg \{\operatorname{det} \boldsymbol{P} \boldsymbol{K}\}+\Delta \arg \left\{\operatorname{det}\left(\operatorname{diag}\{\boldsymbol{P K}\}^{-1}+\boldsymbol{G}\right)\right\}
\end{aligned}
$$

$$
\Delta \arg \{\operatorname{det}(\boldsymbol{I}+\boldsymbol{P} \boldsymbol{K} \boldsymbol{G})\}=\Delta \arg \{\operatorname{det} \boldsymbol{P} \boldsymbol{K}\}
$$$$
+\sum_{i=1}^{m} \Delta \arg \left(1 / \ell_{i}+1\right)+\Delta \arg \operatorname{det}(\boldsymbol{G})
$$$$
-2 \pi\left(N_{C L}-N_{P O}\right)=-2 \pi\left(N_{Z O}-N_{P O}\right)-2 \pi N_{D}
$$

$T$ stable $\Leftrightarrow N_{\mathrm{CL}}=0 \Leftrightarrow N_{\mathrm{ZO}}=-N_{\mathrm{D}}$

\subsection{Input design}

Consider (for example) the transfer of reference signals to the plant input, calculated via the diagonal controller output. This results naturally in non-plant inverting formulas (Boje, 1989).

$$
\begin{gathered}
\left(\boldsymbol{G}^{-1}+\boldsymbol{P K}\right) \boldsymbol{T}_{U^{*} / R}= \\
\left(\boldsymbol{G}^{-1}+\operatorname{diag}\{\boldsymbol{P K}\}\right)(\boldsymbol{I}+\boldsymbol{X}) \boldsymbol{T}_{U^{*} / R}=\boldsymbol{F} \\
\boldsymbol{T}_{U / R}=\boldsymbol{K} \boldsymbol{T}_{U^{*} / R}
\end{gathered}
$$

\subsection{Control authority allocation design}

In MIMO QFT design, the approach is to allocate the specification tolerance by over-bounding the magnitudes of unknown components on the right hand side of relevant design equations by the worstcase specifications. Element-wise this is

Output design

$$
|\boldsymbol{I}-| \boldsymbol{X}|| \boldsymbol{T}_{\text {diag }}|\leq| \boldsymbol{T}|\leq(\boldsymbol{I}+|\boldsymbol{X}|)| \boldsymbol{T}_{\text {diag }} \mid
$$

Input design

$$
\left|\boldsymbol{T}_{U^{*} / R}\right| \leq\left|\boldsymbol{S}_{\text {diag }} \boldsymbol{G} \boldsymbol{F}\right|+|\boldsymbol{X}| \boldsymbol{T}_{U^{*} / R}^{\text {spec. }} \mid,
$$

or

$$
\left|\boldsymbol{T}_{U^{*} / R}\right| \leq\left|\boldsymbol{S}_{\text {diag }} \boldsymbol{G}\right|\left(|\boldsymbol{F}|+\operatorname{od}\{\boldsymbol{P K}\}\left|\boldsymbol{T}_{U^{*} / R}^{\text {spec }}\right|\right)
$$

The control authority allocation problem for this paper is to reduce the interaction index in a systematic way with tolerable use of off-channel effort. This must be done at frequencies where the sensitivity is not small but where loop gain is still required, i.e. in the loop gain cross-over frequency range.

Consider the effect of an elementary operation $\boldsymbol{K}^{\mathrm{n}}$

$$
\left[\boldsymbol{K}^{n}\right]_{i i j j}= \begin{cases}1 & \text { if } \mathrm{ii}=\mathrm{jj} \\ \mathrm{k}_{\mathrm{ij}} & \text { for one } \mathrm{ii}=\mathrm{i}, \mathrm{jj}=\mathrm{j} \\ 0 & \text { otherwise }\end{cases}
$$

on the relevant portion of $\boldsymbol{X}, \operatorname{od}\{\boldsymbol{P K}\} \operatorname{diag}\{\boldsymbol{P K}\}^{-1}$. The $h^{\text {th }}$ row, $j^{\text {th }}$ column element becomes,

$$
\left.\operatorname{od}\{\boldsymbol{P K}\} \operatorname{diag}\{\boldsymbol{P K}\}^{-1}\right]_{h j}=\frac{p_{h j}+p_{h i} k_{i j}}{p_{j j}+p_{j i} k_{i j}} .
$$

This is a linear fractional mapping in $k_{\mathrm{ij}}$ (and hence amenable to design) and the result can be compared to the value before the introduction of $\boldsymbol{K}^{\mathrm{n}}$, $\left[\operatorname{od}\{\boldsymbol{P}\} \operatorname{diag}\{\boldsymbol{P}\}^{-1}\right]_{h j}=\frac{p_{h j}}{p_{j j}}$.

To select which element of $\boldsymbol{X}$ to reduce, notice that the differential sensitivity of $\lambda_{\mathrm{P}}(\mathbf{X})$ with respect to elements of $|\boldsymbol{X}|$, is $\frac{\partial \lambda_{P}}{\partial|x|_{i j}}=\frac{v_{i} w_{j}}{\boldsymbol{v}^{\mathrm{T}} \boldsymbol{w}}>0$ where $\boldsymbol{v}$ and $\boldsymbol{w}$ are the left and right eigenvectors of $|\boldsymbol{X}|$. Note that the Perron root is simple and the eigenvectors can be scaled to be non-negative. The Perron root is therefore analytic and monotonic with respect to $\left|x_{\mathrm{ij}}\right|$.

\section{EXAMPLE}

3.1 Ill conditioned plant-modified benchmark

To illustrate the control authority allocation design, the following 3-input, 2-output example is considered. It is motivated by the 2-input, 2-output a distillation column benchmark problem (Skogestad, Morari and Doyle, 1988, Limebeer, 1991, and references in Boje, 2002a). The original problem is ill conditioned, having an interaction matrix with near unity spectral radius over all frequencies.

Plant:

$$
\boldsymbol{P}(s)=\frac{1}{75 s+1}\left[\begin{array}{ccc}
0.85 & 0.75 & 0.8 \\
1 & 1 & 1
\end{array}\right] \operatorname{diag}\left\{v_{i} e^{-T_{i} s}\right\}
$$

$v_{\mathrm{i}} \in[0.8,1.2] ; T_{\mathrm{i}} \in[0,1]$ minutes representing valve gain uncertainty and input delay respectively

For $\boldsymbol{K}=\left[\begin{array}{lll}1 & 0 & 0 \\ 0 & 1 & 0\end{array}\right]^{\mathrm{T}}$, the Perron root is

$\lambda_{P}\left(\operatorname{od}\{\boldsymbol{P K}\} \operatorname{diag}\{\boldsymbol{P K}\}^{-1}\right)=0.94$ for all frequencies and for all plants (the interaction index is independent of diagonal scaling).

For $\boldsymbol{K}=\left[\begin{array}{lll}1 & 0 & 0 \\ 0 & 0 & 1\end{array}\right]^{\mathrm{T}}$, the Perron root is

$\lambda_{P}\left(\operatorname{od}\{\boldsymbol{P K}\} \operatorname{diag}\{\boldsymbol{P K}\}^{-1}\right)=0.97$.

At design frequency $\omega=0.5 \mathrm{rad} / \mathrm{min}$ (where the original design has big problems with interaction and high sensitivity), allocating control effort from the third input, via $\boldsymbol{K}=\left[\begin{array}{ccc}1 & 0 & k_{31} \\ 0 & 1 & k_{32}\end{array}\right]^{\mathrm{T}}$, allows some improvement in interaction. Figure 2 shows the value 
sets on the plane of $k_{3 j}$ for different levels of improvement (relative to $k_{3 j}=0$ ) and the static allocation indicated, $k_{31}=-5.6 \mathrm{~dB} \angle-180^{\circ}$ and $k_{32}=-6.1 \mathrm{~dB} \angle-180^{\circ}$ result in an interaction index of $\lambda_{P}\left(\operatorname{od}\{\boldsymbol{P K}\} \operatorname{diag}\{\boldsymbol{P K}\}^{-1}\right)=0.92 \approx 0.94 / \sqrt{1.023 \times 1.022}$ For practical engineering, this is hardly an improvement but adequately illustrates the method at a single frequency.

\section{CONCLUSIONS}

This paper has introduced the design of a control authority matrix to precede diagonal feedback controller design in multivariable QFT for overactuated plants. The design approach is to use elementary operations to reduce the interaction at critical frequencies near the cross-over frequency range. If the interaction index can be made less than unity by the combination of the sensitivity design (typically at low frequencies) and forward loop decoupling via control authority allocation (around the gain cross-over frequency) then decentralised stability results apply.

One area for future work is a detailed examination of the possibilities for design of both phase and gain of individual elements to ensure that the reduction in interaction index does not have a negative impact on the location of the squared down system's zeros. There is also scope for application to a realistic problem requiring dynamic allocation and that would exercise practical design constraints such as actuator bandwidth and amplitude limits; cost of control via different inputs and integrity against saturation or actuator failure.

\section{REFERENCES}

Boje E and Nwokah ODI (2001), "Quantitative Feedback Design Using Forward Path Decoupling" ASME, Journal of Dynamic Systems, Measurement and Control, 123, no. 1, 129-132.

Boje E (2002b), "Non-Diagonal Controllers in MIMO Quantitative Feedback Design", International Journal of Robust and Nonlinear
Control, Isaac Horowitz Special Issue (Part 2), 12 , no. 4, 303-320.

Boje E (1989), "Multivariable quantitative feedback designs with plant input specifications", IEEE International Conference on Control and Applications, ICCON 89, Jerusalem, Israel, Session WP-2.

Skogestad S, Morari M and Doyle JC (1988). "Robust control of ill conditioned plants; high purity distillation", IEEE Transactions on Automatic Control, 33, 1092-1105. (See correction, ibid. 1989.)

Boje E (2002a), "Multivariable quantitative feedback design for tracking error specifications", Automatica, 38, no. 1, 131-138.

Nwokah ODI, Nordgren RE and Grewal GS (1995), "Inverse Nyquist Array: A quantitative theory", Proc. IEE, Part D, 142, no. 1, 1995, 23-30.

Limebeer DJN (1982), “The application of generalized diagonal dominance to linear system stability" International Journal of Control, 36, no. 2, 185-212.

Maciejowski JM (1989), Multivariable Feedback Design, Addison Wesley.

Rosenbrock HH (1969), "Design of multivariable control systems using the inverse Nyquist array", Proc. IEE, 116, no. 11, 1929-1936.

Skogestad S and Postlethwaite I (1996), Multivariable Feedback Control, Wiley.

Limebeer DJN (1991). "The specification and purpose of a controller design case study", Proceedings of Conference on Decision and Control, Brighton, pp. 1579-1580. 


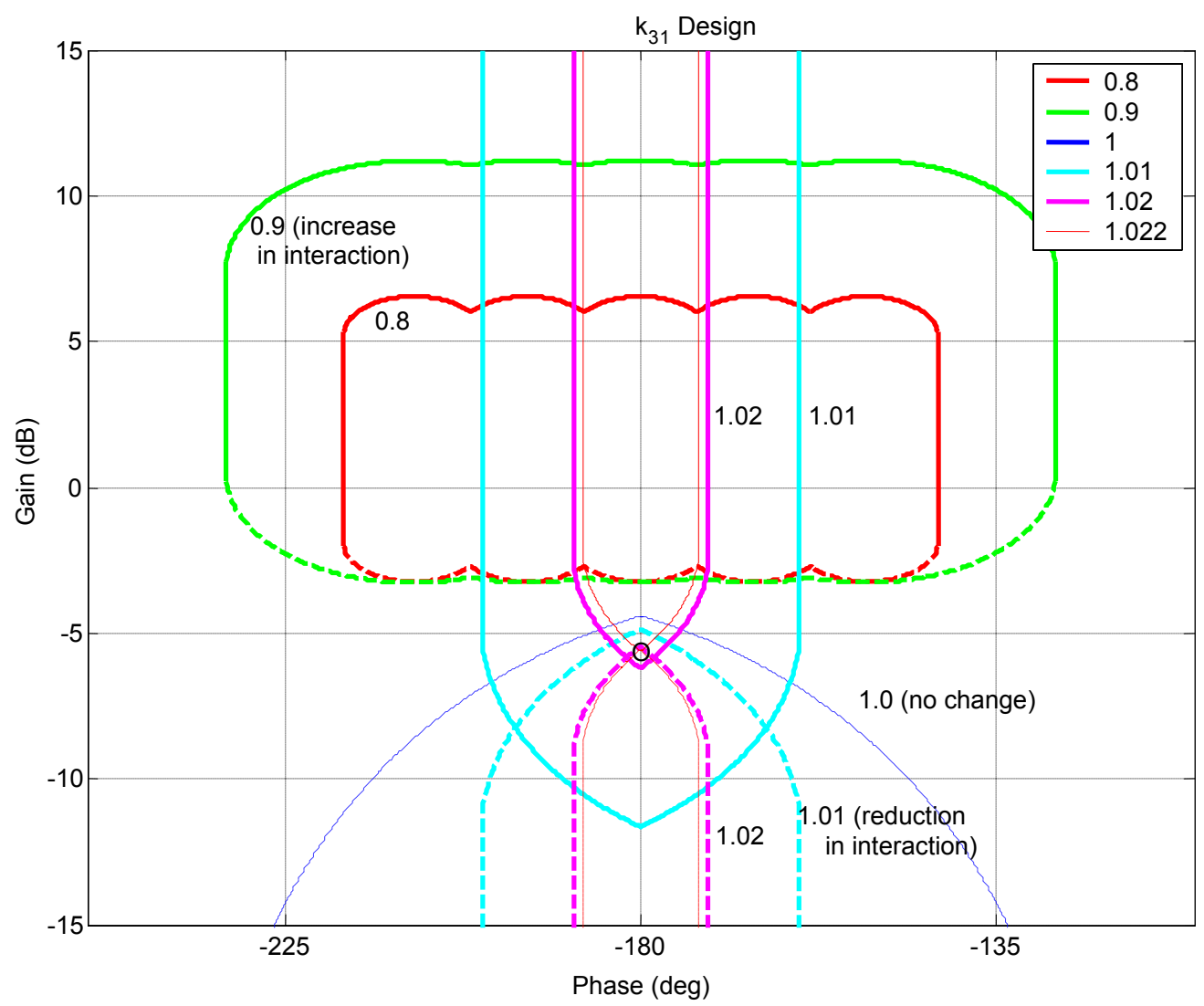

Figure 2a: Design of $k_{31}$

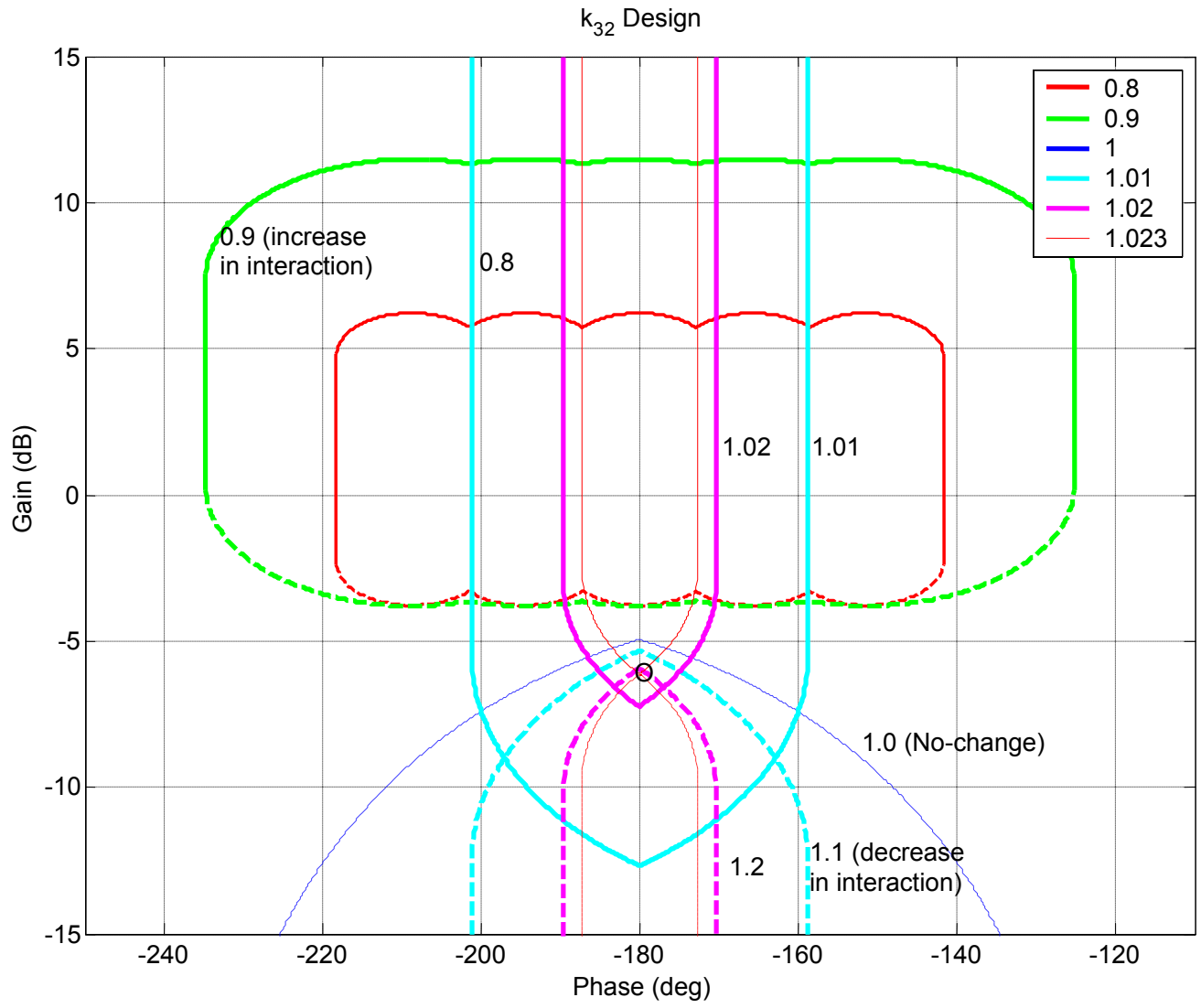

Figure 2b: Design of $k_{32}$ 